\title{
Natural integration of scalar fluxes from complex terrain
}

\author{
John D. Albertson ${ }^{\mathrm{a}, *}$, Marc B. Parlange ${ }^{\mathrm{b}}$ \\ ${ }^{a}$ Department of Environmental Sciences, University of Virginia, Charlottesville, VA 22903, USA \\ ${ }^{\mathrm{b}}$ Department of Geography and Environmental Engineering, The Johns Hopkins University, Baltimore, MD 21218, USA \\ Received 11 June 1998; received in revised form 26 February 1999; accepted 2 March 1999
}

\begin{abstract}
Large eddy simulations of turbulent flow and transport in the atmospheric boundary layer were conducted over heterogeneous sources of heat and water vapor to identify the blending properties of the turbulent mixing in an unstably stratified boundary layer. The numerical simulations show that the concept of blending in the ABL is in fact a useful one, even under convective conditions, for a range of surface conditions. Since the transport eddies that are responsible for the blending have sizes that are constrained by the boundary layer depth, and since the vertical motion is so important under the unstable density stratification studied here, we see that when the length scales of the source variability on the land surface become significantly greater than the ABL depth the blending is lost. In this case the source fields remain relatively uncoupled by the important eddy motion. However, for smaller surface length scales, the dynamic eddy motion couples the surface patches. Hence, there is good reason that the land surface exchange phenomenon would not be scale invariant over the entire range of scales. Because of the active role of temperature the effects of inhomogeneous surface sources of sensible heat persist higher into the ABL than do the effects of surface sources from more passive scalars, such as water vapor. Moreover, the mean fields of potential temperature and specific humidity blend at much lower heights than do the vertical turbulent flux fields of these two scalars. We propose a useful measure of blending efficiency for simulation studies and show how this bridges from the dynamics responsible for the blending to the horizontal homogeneity of scalar flux fields at measurement heights in the ABL. (c) 1999 Elsevier Science Ltd. All rights reserved.
\end{abstract}

Keywords: Land-atmosphere interaction; Evaporation; Turbulence; Surface fluxes

\section{Introduction}

The measurement of water vapor and heat exchange between the land and the atmosphere is an important part of efforts to understand the coupling feedbacks between land surface and atmospheric processes. A common feature of most measurement approaches is the assumption or requirement that the land surface be homogeneous. It is obvious, however, that most land surfaces are far from homogeneous in a strict sense.

Arguments have been made for considering a range of naturally heterogeneous surfaces as being effectively homogenous when sensed from above some height in the turbulent atmospheric boundary layer (ABL) $[31,32]$. The basis for these arguments is that turbulent motion in the ABL is responsible for an extremely efficient mixing of the mass and heat between leaving the distributed surface sources and arriving at some measurement height in the atmospheric surface layer. The

\footnotetext{
${ }^{*}$ Corresponding author. Tel.: +1-804-924-7241; fax: +1-804-9822137; e-mail: jdalbertson@virginia.edu
}

idea goes that we should be able to make measurements through time at some point well above the surface in the $\mathrm{ABL}$ and see the net effect of a large distributed surface area. The height above which measurements sense a statistically homogeneous surface is often referred to as the "blending height". It is well established that this height will depend on the properties of the surface [23]. Typically, the blending height is defined for the mean wind speed and mean scalar quantities, such that measurements of mean profiles above the blending layer provide estimates of net surface fluxes over the regional surface - much as with Monin-Obukhov similarity theory [28] for truly homogeneous surfaces.

In this paper we apply the Large Eddy Simulation (LES) technique to explore the potential for different blending efficiencies for passive versus active scalars (e.g. water vapor versus temperature) and for flux fields versus mean scalar quantities (e.g. sensible heat flux versus temperature).

With respect to mean scalar quantities, fairly well developed theory exists to describe the Internal Boundary Layer (IBL), where the air properties adjust 
over a single patch $[40,33,13,15]$. With a regional patchwork of surface features as addressed here, we are looking at a complicated superposition problem to describe the net effect as a blending layer. Under the IBL definition, the air above this layer is in equilibrium with the upwind patch [15]. Here we study the blending layer, above which the air is in equilibrium with the net regional surface, not just the immediate upwind surface.

Recent experiments have lent observational support to the concept of a blending layer for the mean scalar fields and have demonstrated its usefulness for estimation of regional scale fluxes under both neutral $[7,32]$ and unstable conditions [6,30]. These results are encouraging, however they do not attempt to describe in physical terms the processes that are responsible for the blending nor do they address the question of the blending of fluxes.

Attempts have been made to describe this blending process for mean fields with semi-analytical solutions and Reynolds-averaged modeling tools $[9,41,34]$. These efforts have yielded results for blending of mean variables, largely employing diffusion-analogy solutions as a proxy for the turbulence. The blending of turbulent flux fields does not submit readily to simple modeling techniques nor is it easily measured. The Reynolds-averaged modeling and semi-analytic solutions to diffusion equations can not capture the non-local transport features that can be so important for mixing over strongly heterogeneous surfaces. These approaches solve for mean fields using calibrated models to account for the effect of the turbulent flux fields on these mean fields. In this study it is the turbulent flux fields which we wish to explore. Hence, we avoid the use of a priori decisions for how these fields behave.

Here we explore the dynamics behind this blending phenomenon in order to move toward a physical understanding. This should ultimately support the development of a relationship which describes or predicts the depth of the blending layer in terms of certain large scale surface and flow properties. In this paper we explore this problem through simulations of turbulent transport of water vapor and heat over and from the land surface. We employ the LES technique, where the turbulent flow and mixing are observed directly from numerical integrations of the Navier-Stokes equations through space and time over a complex land surface. The conditions studied here are extreme, with strong differences in the strength of convective forcing along the longitudinal axis of the land surface. These conditions were chosen to present a difficult set of cases in which to test the efficacy of the blending mechanisms.

We first present a brief description of the LES technique and discuss our code and the numerical experiments that were conducted. Finally, we present the results of the numerical simulations and the observations of blending with a description of the processes and inference of governing variables.

\section{Large Eddy Simulation}

The turbulent flow in the ABL is described by the Navier-Stokes equations written for a wall bounded flow subject to thermal stratification [19]. These equations describe dynamics occurring over a continuous range of scales from $\mathrm{O} 10^{-3}$ to $\mathrm{O} 10^{3} \mathrm{~m}$. Moreover, these six decades of scale are active on each of the three spatial dimensions and are nonsteady in time (i.e. $10^{18}$ degrees of freedom at each instant in time). With state of the art computational techniques we are limited to dealing with no more than say $10^{6}-10^{9}$ computational nodes in space, depending on the particular system. A popular approach over the past several decades has been to ensemble average the governing equations and solve for the mean fields with the net effects of the entire turbulent fields approximated by some closure model (See review by Launder [20]). However, with the so-called Reynolds Averaged approach, the closure model must be carefully calibrated against experimental data and the modeler is well advised not to attempt to apply the model to a set of conditions outside those for which it was originally calibrated. The later approach is not well suited to the present problem, where the dynamics and mixing of the turbulent field is precisely what we wish to study. Moreover, the Reynolds Averaged approach is notoriously ill-equipped to deal with flow situations such as the one we study here, where non-local transport (i.e. not proportional to local gradients) is important. In simple terms, we can not at once average out the turbulence and still explore its dynamical interactions.

With the LES technique we explicitly simulate the large scale turbulent field (e.g. eddies larger than a few meters) and model the effects of the small scale eddies, which are less important to transport processes and by virtue of their locally isotropic structure submit more readily to simple parameterization. The large scales of motion are separated from the small scales of motion through the explicit convolution of a filter over the fields of interest [21]. The large scale eddies are "simulated", with the effects of the small scale eddies "modeled" by a subgrid scale (SGS) model. The LES technique was pioneered in the early 1970s by Deardorff [10-12] and has become a popular tool for studying engineering and geophysical turbulent flows [14]. It is a natural approach for studying land-atmosphere interaction, for it is the large scales that are both responsible for carrying the flux and most affected by the boundary conditions.

Several LES applications have demonstrated the ability of the technique to reproduce canonical turbulence profiles in the horizontally homogeneous ABL $[25,26,4,29]$. Several other recent applications have 
explored the effect of observed heterogeneous surface conditions on overall boundary layer development $[18,36,5]$. This paper extends the use of LES into an exploration of blending layers over patchy surfaces with distinct and abrupt transitions in surface temperature, wetness, and roughness.

The base version of our LES code, which simulates velocity fields and momentum transport in a neutrally stratified boundary layer, was described earlier by Albertson [1] and Albertson and Parlange [2]. Here it is extended to include the transport of temperature and water vapor, the buoyancy effects these scalars have on the momentum equations, and several other changes as needed to capture the role of a capping inversion. A brief description of the LES equations and their numerical solution follows.

\subsection{Basic equations}

The velocity field is governed by filtered equations describing the conservation of mass for an incompressible flow

$\partial_{i} \bar{u}_{i}=0$

and the conservation of momentum, under the Boussinesq approximation,

$\partial_{o} \bar{u}_{i}+\overline{\bar{u}_{j}\left(\partial_{j} \bar{u}_{i}-\partial_{i} \bar{u}_{j}\right)}=-\partial_{i} \bar{p}+F_{p} \delta_{i 1}-\partial_{j} \tau_{i j}+\bar{\beta} \delta_{i 3}$,

where $\bar{u}_{i}$ is the resolved (i.e. filtered) velocity component in the $x_{i}$ direction $(i=1,2,3), \bar{p}$ is a dynamic pressure term (which includes the trace of the subgrid stress tensor and the $x_{i}$ direction gradient of the turbulent kinetic energy), $F_{\mathrm{p}}$ is the mean streamwise pressure gradient (forcing), $\tau_{i j}$ is the subgrid stress tensor, $\bar{\beta}$ is the buoyancy parameter (described below), $\partial_{o}$ is the time derivative, $\partial_{k}$ is a spatial derivative with respect to direction $x_{k}$, and $\delta_{i j}$ is the Kronecker delta ( 1 for $i=j, 0$ for $i \neq j$ ). The turbulent pressure field is defined at each time step by the application of Eq. (1) to Eq. (2) and solving the resulting Poisson equation. Note that we use the overbar to denote a resolved or filtered field, not to be confused with the traditional ensemble average field. Hence, $\bar{u}_{i}$ varies in space and time according to the large scale eddy motion.

The effect of the subgrid stresses on the resolved eddy motion is approximated as a local, subgrid eddy-viscosity acting on the resolved instantaneous gradients [38]

$\tau_{i j}=-2 v_{T} S_{i j}$,

where $S_{i j}\left(=1 / 2\left(\partial_{i} \bar{u}_{j}+\partial_{j} \bar{u}_{i}\right)\right)$ is the resolved strain rate tensor and $v_{T}\left(=\left(c_{\mathrm{s}} l\right)^{2} \sqrt{2 S_{i j} S_{i j}}\right)$ is the eddy viscosity, which varies in space and time with the strain rates, as well as potentially with the Smagorinsky constant $\left(c_{\mathrm{s}}\right)$ and the length scale of the filter $(l)$. The implied summation on the repeated subscripts in this Einsteinian tensor notation renders this eddy-viscosity a scalar field.
The resolved scalar fields are also governed by filtered conservation equations

$\partial_{o} \bar{\theta}+\overline{\bar{u}_{j} \partial_{j} \bar{\theta}}=-\partial_{j} \pi_{j}^{\theta}$,

$\partial_{o} \bar{q}+\overline{\bar{u}_{j} \partial_{j} \bar{q}}=-\partial_{j} \pi_{j}^{q}$,

where $\bar{\theta}$ is the filtered potential temperature field, $\bar{q}$ is the filtered humidity field, and $\pi_{j}^{\theta}$ and $\pi_{j}^{q}$ are the subgrid fluxes of temperature and water vapor, respectively, in the $x_{j}$ direction. As with the momentum field, these subgrid fluxes are approximated from a local eddy-viscosity model

$\pi_{j}^{\theta}=-\frac{v_{T}}{S_{c}} \partial_{j} \bar{\theta}$,

$\pi_{j}^{q}=-\frac{v_{T}}{S_{c}} \partial_{j} \bar{q}$,

where $S_{c}$ is a general scalar Schmidt number, representing the relative efficiencies of transport of momentum and scalar fields.

Turbulent fluctuations in the temperature and humidity field create vertical accelerations in the momentum field, through the buoyancy term, $\bar{\beta}$. Following the Boussinesq approximation, it is the normalized deviations (from the horizontal average) in the virtual potential temperature that create these density driven accelerations, i.e.

$\bar{\beta}(x, y, z, t)=\frac{\left(\bar{\theta}_{v}(x, y, z, t)-\tilde{\bar{\theta}}_{v}(z, t)\right)}{\tilde{\bar{\theta}}_{v}(z, t)} g$,
$\tilde{\bar{\theta}}_{v}(z, t)=\frac{1}{L_{x} L_{y}} \iint \bar{\theta}_{v}(x, y, z, t) \mathrm{d} x \mathrm{~d} y$,

where $\bar{\theta}_{v}=\bar{\theta}+0.61 \bar{q}$ (with temperature in $[\mathrm{K}]$, water vapor concentration in $[\mathrm{g} / \mathrm{km}]$, and the constant $(0.61)$ clears units), $g$ is the acceleration due to gravity, and the $\sim$ is to define a horizontal average. For most conditions the bulk of $\bar{\beta}$ is due to the potential temperature fluctuations, with a minor contribution from the water vapor field. See Stull [39] (pp. 81-85) for an excellent presentation of the Boussinesq approximation and buoyancy driven accelerations.

\subsection{Boundary Conditions}

The horizontal directions are assigned periodic boundary conditions, such that for any primitive flow variable, $A$, we have $A\left(x+m L_{x}, y+n L_{y}, z\right)=A(x, y, z)$, where $L_{x}$ and $L_{y}$ are the domain dimensions in the $x$ and $y$ direction, and $m$ and $n$ are signed integers. This condition is a great help in turbulence simulation because it is necessary to have fully developed turbulence on both the inflow and exit faces of the domain. However, it is important to prescribe a domain size that is physically large enough to ensure that the flow becomes completely uncorrelated as it passes across the domain $L_{x}$ from inlet 
to exit. Typically, the domain length is kept larger than twice the spatial lag at which the velocity autocorrelation function vanishes. With the present applications this criterion is well exceeded, as the domain length is $>6 \mathrm{~km}$.

The top boundary of the computational domain is positioned well above the top of the boundary layer (500 $\mathrm{m}$ above), defined as the location of the steep stable capping inversion. It is assigned conditions of vanishing vertical gradients of all primitive variables and no flow through the boundary (i.e. $w=0$ ). A numerical sponge is added to the vertical momentum equation to dampen vertical motions as they approach the no-flow upper boundary [29]. The effects of this dampening are limited to a small layer located well above the capping inversion, and hence isolated from the important boundary layer dynamics.

The bottom boundary is far more critical to the structure of the turbulence than the sides and top. This is because the mechanical turbulence is produced as a result of drag at the wall, and the heat flux responsible for the convective motion is introduced at the wall as well. The exchange of scalars and momentum between the flow and the surface are affected by instantaneous exchange equations that depend on the local differences between the properties of the surface (prescribed fields) and the air just overlying that location at that point in time. For a general scalar flux $\left(f_{\mathrm{s}}\right)$ we write this as

$$
\begin{gathered}
f_{\mathrm{s}}(x, y, t)=\left(s_{o}(x, y, t)-\bar{s}(x, y, \Delta z / 2, t)\right) \sqrt{-\tau_{o}} k \\
{\left[\ln \left(\frac{\Delta z / 2}{z_{\mathrm{os}}}\right)\right]^{-1},}
\end{gathered}
$$

where $s_{o}(x, y, t)$ is the local surface value (concentration) of the scalar, $\bar{s}(x, y, \Delta z / 2, t)$ is the scalar value at the first node above the surface (note that as the grid is staggered, the first scalar node is at $\Delta z / 2), \tau_{o}$ is the instantaneous local surface shear stress, and $z_{\mathrm{os}}$ is the scalar roughness length. Since the simulations that we study here represent experiments on the order of an hour in duration, the prescribed spatial fields of surface temperature and wetness fields are held constant in time $\left(s_{o}(x, y, t) \rightarrow s_{o}(x, y)\right)$. We note that, in the field, surface temperature and wetness fields are evolving during the day. However, this assumption of static surface conditions may be considered representative of mid-day conditions, bracketing solar-noon. Furthermore, the presence or lack of a modest trend in the surface temperature and wetness would most likely not have noticeable impacts on the results described here. Although the type of surface exchange formulation used in Eq. (7) has its roots as a relationship between mean values, it has been extended to instantaneous values in boundary layer simulations in the past $[25,24,37]$. We envision its use as a wall-specific subgrid model which relates instantaneous resolved fields to local instantaneous exchange rates.

Typically, LES codes use specified surface fluxes (rather than specified surface concentrations), but such an approach provides a horizontal decoupling of the surface fluxes over heterogeneous source fields, since local air properties will not affect the fluxes. The use of Eq. (7) captures these spatially coupled feedbacks, where the flux $\left(f_{\mathrm{s}}\right)$ affects the local air properties, which advect downwind and in turn affect the downwind distributions of $f_{\mathrm{s}}$.

The turbulent pressure field receives periodic horizontal boundary conditions and vertical boundary conditions from considerations of the vertical momentum equation written at the wall and the top of the domain.

\subsection{Numerical Technique}

With periodic boundary conditions in the $x$ and $y$ directions the resolved flow variable fields (e.g. $\bar{A}(x, y, z)$ ) may be transformed horizontally into periodic functions for numerical treatment

$\bar{A}(x, y, z)=\sum_{k_{x}} \sum_{k_{y}} \hat{A}\left(k_{x}, k_{y}, z\right) \mathrm{e}^{\mathrm{i}\left(k_{x} x+k_{y} y\right)}$,

where $\hat{A}$ is the complex amplitude of the 2-D Fourier transform, $k_{x}$ and $k_{y}$ are the wavenumbers in the $x$ and $y$ directions, respectively, the summation is over all resolved wavenumbers, $-N_{x} / 2+1 \leqslant k_{x} \leqslant N_{x} / 2$ and $-N_{y} / 2+1 \leqslant k_{y} \leqslant N_{y} / 2$, and $\mathrm{i}\left(=(-1)^{1 / 2}\right)$ is the imaginary number. For this 2-dimensional transform it is understood that $x$ and $y$ assume values constrained to the quadrature points $x=2 \pi(\mathrm{i}-1) / N_{x}$ and $y=$ $2 \pi(\mathrm{j}-1) / N_{y}$. The transform in Eq. (8) is invertible (i.e. $\bar{A} \Longleftrightarrow \hat{A}$ ) since it is linear and complete in the sense of a Hilbert space [8]. All the resolved fields are filtered such that they are fully resolved by the range of modes included in this transform. Hence, the filtering is essentially a low pass filter.

In the present LES code the numerics are mixed, with a Fourier-based pseudospectral approach in the horizontal directions and finite difference in the vertical (after Moin et al. [27]). The Horizontal derivatives come from a straight application of the spatial derivative operator to Eq. (8), i.e.

$$
\begin{aligned}
& \partial_{x} \bar{A}(x, y, z)=\sum_{k_{x}}^{\prime} \sum_{k_{y}}^{\prime}\left[\hat{A}\left(k_{x}, k_{y}, z\right) \mathrm{i} k_{x}\right] \mathrm{e}^{\mathrm{i}\left(k_{x} x+k_{y} y\right),} \\
& \partial_{y} \bar{A}(x, y, z)=\sum_{k_{x}}^{\prime} \sum_{k_{y}}^{\prime}\left[\hat{A}\left(k_{x}, k_{y}, z\right) \mathrm{i} k_{y}\right] \mathrm{e}^{\mathrm{i}\left(k_{x} x+k_{y} y\right),}
\end{aligned}
$$

where the primed summation operators are over all resolved wavenumbers, except the Nyquist (i.e. $N / 2$ ).

The vertical direction is not periodic and is, therefore, treated with conventional second-order accurate finite differences. 
The time advancement is by a second-order accurate Adams-Bashforth routine (Canuto et al. [8], p. 102), which is popular for turbulent flows as it is accurate [16] and tends to have a dampening effect on any oscillatory computational mode (Haltiner and Williams [17], p. 151).

The potential for aliasing effects, where high wavenumber energy is folded back to resolved scales from the physical-space product in the convective terms, is reduced by a partial dealiasing [1].

\section{Numerical Experiments}

To explore the blending of heterogeneous surface fluxes up into the $A B L$, we performed systematic $3 \mathrm{D}$, transient turbulent flow and transport simulations over several different surfaces. As our primary objectives are to define the characteristics of this blending and begin to isolate large scale flow variables that control the height to which the effects of the surface heterogeneities persist into the ABL (i.e. the depth of the blending layer), we simulate flow over patchy surfaces, with the length scale of the patches changing from simulation to simulation. As a benchmark in the intercomparison of these cases we employ a base-case with a completely homogenous surface, but with similar spatially averaged surface fluxes as in the heterogeneous cases. Relative to this base case, we study cases with surface patch lengths $\left(L_{\mathrm{s}}\right)$ that are:

(i) significantly smaller than the ABL depth $(N=16$ patches and $L_{\mathrm{s}}=393 \mathrm{~m}$ );

(ii) on the order of the ABL depth $(\mathrm{N}=8$ and $L_{\mathrm{s}}=785 \mathrm{~m}$ );

(iii) significantly greater than the ABL depth $(N=2$ and $L_{\mathrm{s}}=3142 \mathrm{~m}$ ).

The reasoning behind these selections is related to the effect the depth of the boundary layer has in constraining the upper size of the large scale transport eddies. Therefore, we look at a case where the surface features are smaller than the largest eddies, a case where they are of similar scale, and a case where the surface features are significantly larger. We then study how the mixing (or blending) across these patches behaves in each case.

The general features that are common to all cases (homogeneous and heterogeneous) are: The approximate depth of the ABL is $z_{i}=1000 \mathrm{~m}$; The size of the simulated flow domain is $L_{x}=L_{y}=6283 \mathrm{~m}$ (i.e. $2 \pi z_{i}$ ) and $L_{z}=1500 \mathrm{~m}$; The number of simulation nodes is $N_{x}(=64) * N_{y}(=32) * N_{z}(=80)=163,840$; and the initial mixed layer state has a temperature of $25^{\circ} \mathrm{C}$ and a specific humidity of $10 \mathrm{~g} / \mathrm{kg}$. In all cases the average friction velocity is $0.3 \mathrm{~m} / \mathrm{s}$, and the average surface flux of sensible heat is of order $200 \mathrm{w} / \mathrm{m}^{2}$.

The heterogeneous surface cases are designed to simulate flow over an alternating patchwork of arid fields (hot, dry, and smooth) and freely evaporating vegetated areas (cool, wet, and rough). The patches vary only in the direction of the mean wind, such that they appear as stripes of well defined width in the streamwise direction, but which continue infinitely in the transverse direction. An important aspect of these cases is that the buoyant forcing changes drastically between the arid and wet patches. This poses a difficult case for the blending mechanisms.

The arid fields are defined as having a momentum roughness length of $15 \mathrm{~cm}$, a surface temperature of $37^{\circ} \mathrm{C}$, and a surface specific humidity of $11.0 \mathrm{~g} / \mathrm{kg}$. The vegetated patches are defined with a momentum roughness length of $75 \mathrm{~cm}$, a surface temperature of $27^{\circ} \mathrm{C}$, and a surface specific humidity of $13.0 \mathrm{~g} / \mathrm{kg}$. The homogeneous surface, base case, is defined to be an effective average of the arid and well-watered surfaces. Hence, the base case has a momentum roughness length of $34 \mathrm{~cm}$ (from log-average), a surface temperature of $32^{\circ} \mathrm{C}$, and a surface specific humidity of 12.0 $\mathrm{g} / \mathrm{kg}$.

\section{Results and Discussion}

Large eddy simulations of ABL flow over complex terrain generate vast amounts of information, representing all the velocity components and scalars through three space dimensions and the full time duration of the simulation. Hence, appropriate statistical measures must be defined, quantified, and analyzed to address the specific objectives. In this study we address the turbulent mixing of fluxes from patchy terrain, and hence we focus directly on interaction of the scalar fields and the vertical velocity field.

\subsection{LES output and synthesis}

Fig. 1 shows three alternative views of the interaction of vertical velocity and water vapor concentrations above the land surface for the case with eight surface patches $\left(L_{\mathrm{s}}=785 \mathrm{~m}\right)$. We use the water vapor transport from this surface case as an example for exploring the statistical measures that we will employ for quantifying the atmospheric response to surface heterogeneity. We then compare the three cases, relative to the homogeneous case. The top panel of Fig. 1 depicts a sample of the instantaneous vertical latent heat flux along a twodimensional slice oriented in the vertical along the mean wind direction. The yellow regions represent large coherent eddies carrying water vapor up through the ABL. In the middle panel we see a time averaged image of the vertical latent heat flux over this vertical slice. The fluxes are averaged over approximately $45 \mathrm{~min}$ in this image, thus removing the random nature of the flow to show the persistent effects of the surface on the ABL. To 

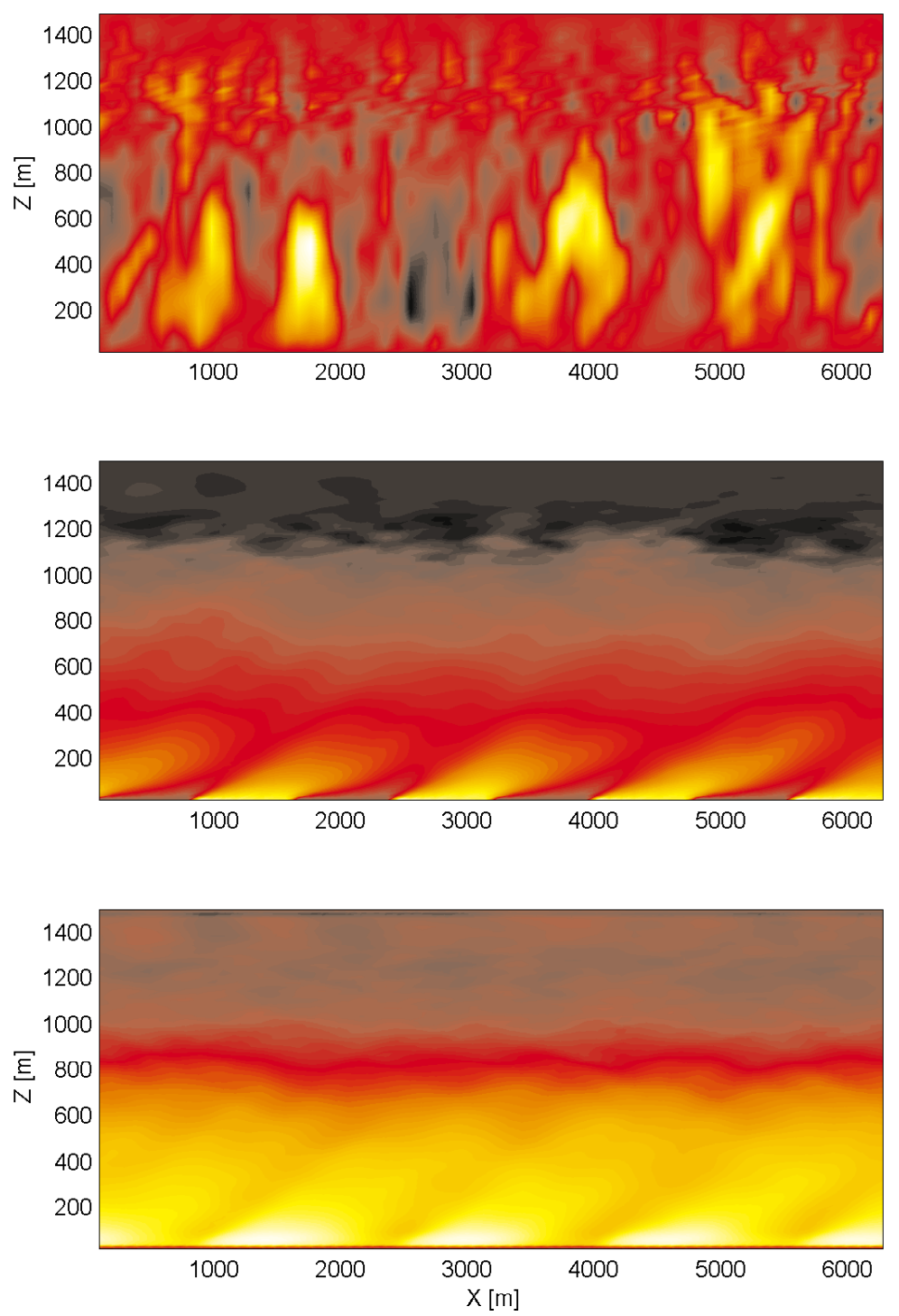

Fig. 1. Top panel: a vertical cross section of the instantaneous vertical latent heat flux; middle panel: vertical cross section of the time averaged vertical latent heat flux; bottom panel: vertical cross section of the correlation coefficient between vertical velocity and specific humidity. Note that this is the case with eight patches, each of length $785 \mathrm{~m}$ in the longitudinal direction, and that the yellow regions are wet and the red dry.

further refine our picture of how the surface affects the dynamical interaction between the velocity and scalar fields we explore in the bottom panel of Fig. 1 the correlation coefficient between vertical velocity and water vapor fluctuations.

To examine the relationships between these images and to place this in the context of the traditional hydrometeorlogical nomenclature, we provide the following relationships. The instantaneous vertical latent heat flux is by definition

$L E(x, y, z, t)=\rho_{\mathrm{a}} L_{\mathrm{v}} w(x, y, z, t) q(x, y, z, t)$, where $\rho_{\mathrm{a}}$ is the density of air, $L_{\mathrm{v}}$ is the latent heat of vaporization, and $w$ and $q$ are the local instantaneous values of the vertical wind speed and specific humidity.
Expanding these fields into local time averages $(\langle\cdot\rangle)$ and fluctuations (") from the time averages we have the identity

$$
\begin{aligned}
& L E(x, y, z, t)=\rho_{\mathrm{a}} L_{\mathrm{v}}\left(\langle w(x, y, z)\rangle+w(x, y, z, t)^{\prime \prime}\right) \\
& \left(\langle q(x, y, z)\rangle+q(x, y, z, t)^{\prime \prime}\right) .
\end{aligned}
$$

This instantaneous flux is what is shown in the top panel of Fig. 1. Note that the fluctuations from the time averages shown here (double-primes) are comprised in the LES of resolved (over bar) plus subgrid (single prime) components. Time averaging of Eq. (11), as needed to obtain the field in the middle panel of Fig. 1, yields 


$$
\begin{aligned}
\langle L E(x, y, z)\rangle= & \rho_{\mathrm{a}} L_{\mathrm{v}}\left(\left\langle w(x, y, z)^{\prime \prime} q(x, y, z)^{\prime \prime}\right\rangle\right. \\
& +\langle w(x, y, z)\rangle\langle q(x, y, z)\rangle),
\end{aligned}
$$

where for horizontally homogeneous flow in a boundary layer the second term on the right must vanish (as $\langle\mathrm{w}\rangle=0$ ), and the mean latent heat flux is proportional to the covariance between $w$ and $q$ (hence, Reynolds averaging rules apply here). The covariance normalized by the local temporal standard deviations of the two variables $\left(\sigma_{w}\right.$ and $\left.\sigma_{q}\right)$ yields the correlation coefficient

$\rho_{w q}(x, y, z)=\frac{\left\langle w(x, y, z)^{\prime \prime} q(x, y, z)^{\prime \prime}\right\rangle}{\sigma_{w}(x, y, z) \sigma_{q}(x, y, z)}$.

The same relationships hold for the sensible heat flux, with $L E$ replaced by $H, q$ replaced by the potential temperature $\theta$, and $L_{\mathrm{v}}$ replaced by the specific heat capacity of air at constant pressure $c_{\mathrm{p}}$.

\subsection{Blending measures}

Before presenting the induced spatial patterns in the correlation coefficients for the different cases, we examine further the vertical structure of the ABL's response to this case, where surface patch length is on the order of (but somewhat smaller than) the ABL depth. Specifically, we are interested in using the LES to examine how the turbulent flow blends over the patchy surface, and renders the flow more horizontally homogeneous with greater heights into the ABL. We offer several views of this. First, in Fig. 2, we look at the time averaged vertical structure of specific humidity, potential temperature, latent heat flux, and sensible heat flux conditionally averaged over each of the two surface types. Note that this figure focuses on the ABL portion of the computational domain, whereas the upper boundary is imposed well above this layer at $z=1500 \mathrm{~m}$. The solid lines represent a piece-wise horizontal average of the time averages above the well-watered surface regions, and the dotted lines represent similar averaging but above the arid surface regions. In the bottom two panels it is evident that the magnitude of the sensible heat flux over the arid regions is comparable in magnitude to the latent heat flux over the wet, vegetated regions. Similarly, the low heat flux values are also comparable. Note that the prescribed surface temperature differences and water vapor concentration differences alone would not give these comparable fluxes, but that when taken together with the roughness differences of the two surface types we achieve similar heat flux maxima and minima. Interestingly, Fig. 2 shows that the mean fields are less strongly affected by the surface heterogeneity than are the flux profiles. A problem with this sort of presentation is that the transport features of the flow cause upper regions of the boundary layer over one type patch to be perhaps more affected by an upwind patch than by the surface immediately under the location of interest. This can be seen in Fig. 1. Hence, the conditional averaging is most useful in the lower portions of the ABL. Note from
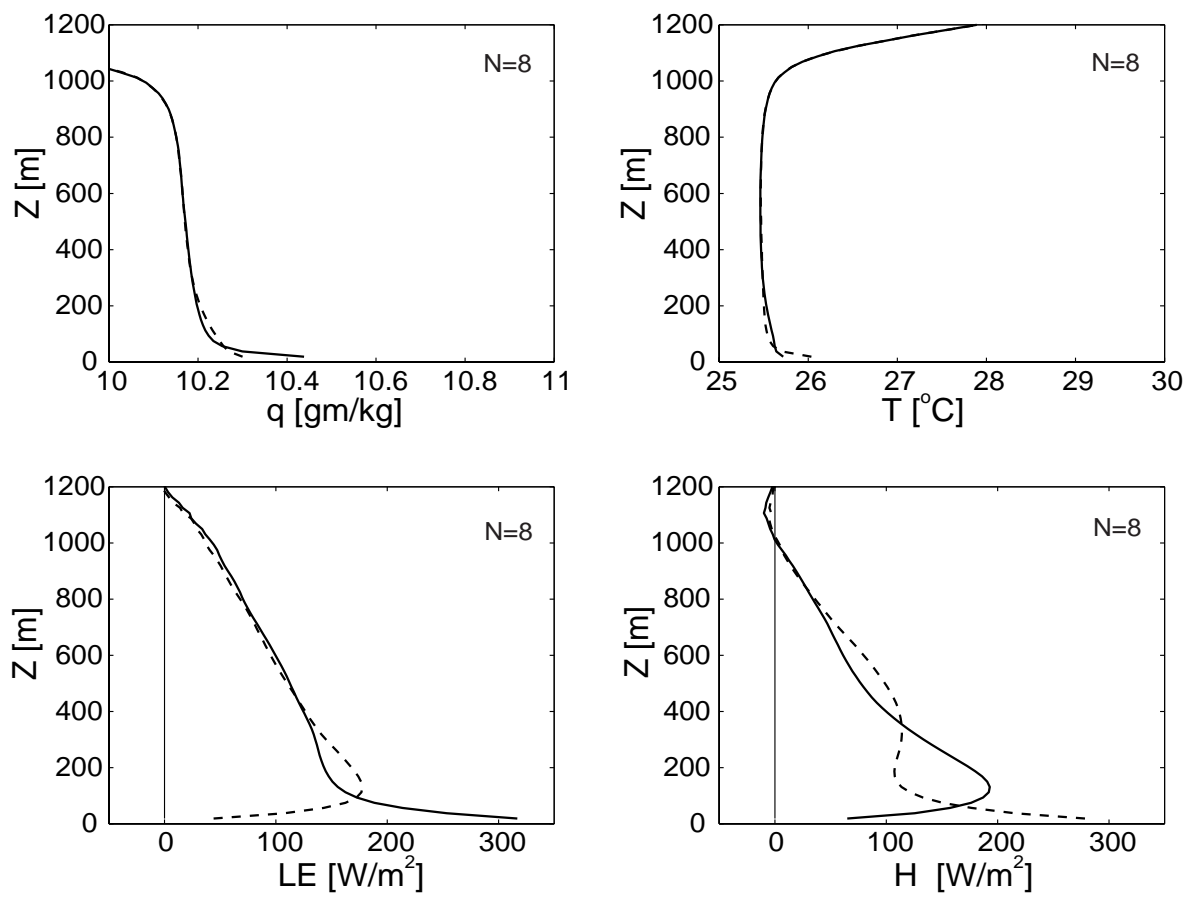

Fig. 2. Time averaged vertical profiles of specific humidity, potential temperature, latent heat flux, and sensible heat flux, conditionally averaged over each of the two surface types. The solid lines represent a piece-wise horizontal average above the well-watered surface regions, and the dotted lines represent averaging above the arid surface regions. 
Fig. 2, though, how the complete horizontal averages (average of two lines) conforms qualitatively to what one would expect for a homogeneous ABL. It is also apparent that eddy correlation fluxes measured at two different heights above the blending layer, over either patch type, could be extrapolated by a straight line to give reliable estimates of the regional scale average surface fluxes. This is in keeping with the concept of a blending layer as defined in the Introduction.

In Fig. 3 we see a more general view of the blending of the mean fields and fluxes with height in the ABL. The measure shown here is basically $(+$ and -$)$ the horizontal standard deviation of the time averages, plotted versus height. If $A(x, z)$ is the time (and $Y$ ) averaged field, this measure is defined as

$$
\begin{aligned}
& \Delta A(z)=\left(\frac{1}{L_{x}} \int[A(x, z)-\tilde{A}(z)]^{2} \mathrm{~d} x\right)^{1 / 2}, \\
& \tilde{A}(z)=\frac{1}{L_{x}} \int A(x, z) \mathrm{d} x .
\end{aligned}
$$

This shows clearly how the flux profiles and mean scalar profiles become more horizontally homogeneous at greater heights. Note the key results that, in general, the means blend lower than do the fluxes, and the latent heat flux blends lower than the sensible heat flux. We discuss this in greater detail later. It is important to keep in mind that these profiles begin at a height of $\Delta z / 2(=9$ $\mathrm{m})$ above the ground. Between this height and the ground surface the scalar profiles move from these small differences to the large differences in the prescribed values at the surface (e.g. difference of $10^{\circ} \mathrm{C}$ for temperature).

We now turn to focus on the induced spatial patterns in the correlation coefficients between vertical velocity and water vapor concentrations $\left(\rho_{w q}\right)$ and between vertical velocity and potential temperature $\left(\rho_{w \theta}\right)$. These normalized measures of the fluxes (c.f. Eq. (13)) offer a more refined picture of the turbulent interactions, as they remove effects on the fluxes due to spatial patterns in the variances. Also the correlation coefficients decrease less quickly with height than do the fluxes, which decrease strongly due to a product of the decreasing correlation coefficients and the decreasing variances with height in the mixed layer.

\subsection{Effects of surface patchiness on vertical transport}

The effect of the surface variability on $\rho_{w q}$ in the ABL is shown for the three different heterogeneous cases in Fig. 4, with the large patches in the top panel showing persistent effects through the full the depth of the boundary layer and the smallest patches in the bottom panel affecting only the lower region of the boundary layer. A similar presentation is provided for $\rho_{w \theta}$ in Fig. 5. From a comparison of Figs. 4 and 5 it is apparent that the effects of the surface temperature heterogeneities persist much higher into the ABL than the effects of surface moisture heterogeneities, which is responsible for the differences in latent and sensible heat blending shown in Fig. 3. The surface fluxes are of
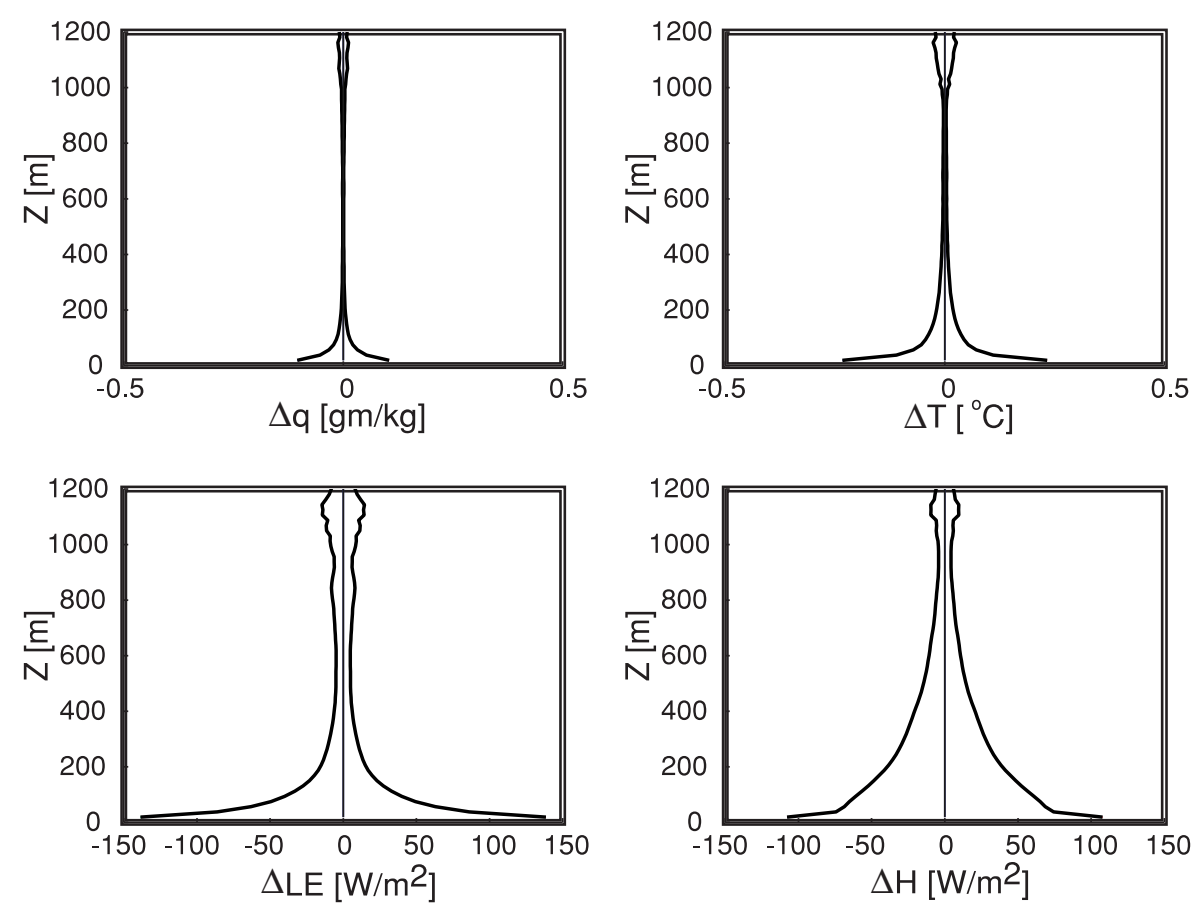

Fig. 3. Vertical profiles of the horizontal variability of the specific humidity, potential temperature, latent heat flux, and sensible heat flux ( + and - the horizontal standard deviation of the time averages of each of these variables). 

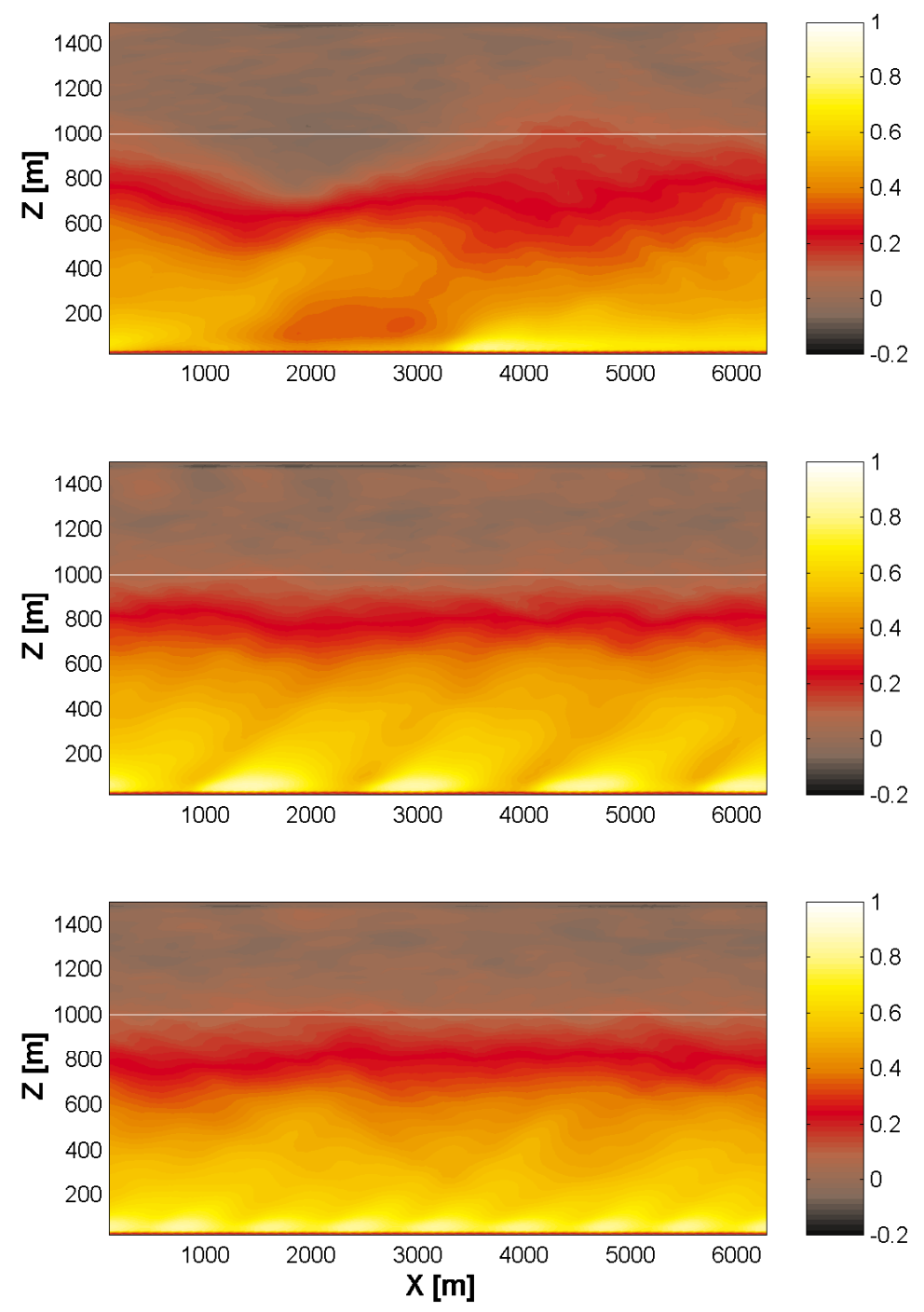

Fig. 4. A comparison of the vertical cross sections of correlation coefficient between vertical velocity and specific humidity $\left(\rho_{w q}\right)$ for the three heterogeneous surface cases. This measure is averaged in time and transverse $(Y)$, and shown versus longitudinal $(X)$ and vertical $(Z)$ axes. The top, middle, and bottom panels show $\rho_{w q}$ over surfaces with patch lengths $\left(L_{\mathrm{s}}\right)$ of 3142,785 , and $393 \mathrm{~m}$, respectively.

similar magnitude (mean and difference between two surfaces) for both sensible and latent heat. Hence, the magnitude of the sensible heat flux from the arid surface is similar (in $\mathrm{W} / \mathrm{m}^{2}$ ) to the latent heat flux from the well watered vegetation surface, and the latent heat flux from the arid surface is similar to the sensible heat flux from the well watered vegetation surface. The blending appears to be more efficient for latent heat flux than for sensible heat, under similar surface variability. This is most likely due to the active role of temperature on the vertical velocity (c.f. Eqs. (2) and (6)), whereby the temperature fluctuations induce vertical accelerations, which in turn carry the temperature fluctuations to greater heights in a coherent parcel of air. Water vapor, on the other hand, is much more passive and tends to be carried simply by the velocity field, with minimal feedback. Another way to consider this point is that the high sensible heat fluxes have a much more pronounced effect on the convective motion than do the otherwise comparable high latent heat fluxes. In fact, any measure of ABL stability would vary dramatically in $X$ below the blending height.

\subsection{Effects of surface patchiness on scalar similarity}

The interactions between the different scalar sources are shown in Fig. 6, which presents the correlation coefficient between potential temperature $(\theta)$ and water vapor $(q)$ versus $X$ and $Z$ for the three heterogeneous cases. For the largest surface features (top panel) we see the strongest correlations between $\theta$ and $q$ over the hotdry patch, implying that water is being carried horizontally from the wet to the dry patch, where it is swept vertically in the convective motion driven by the large 

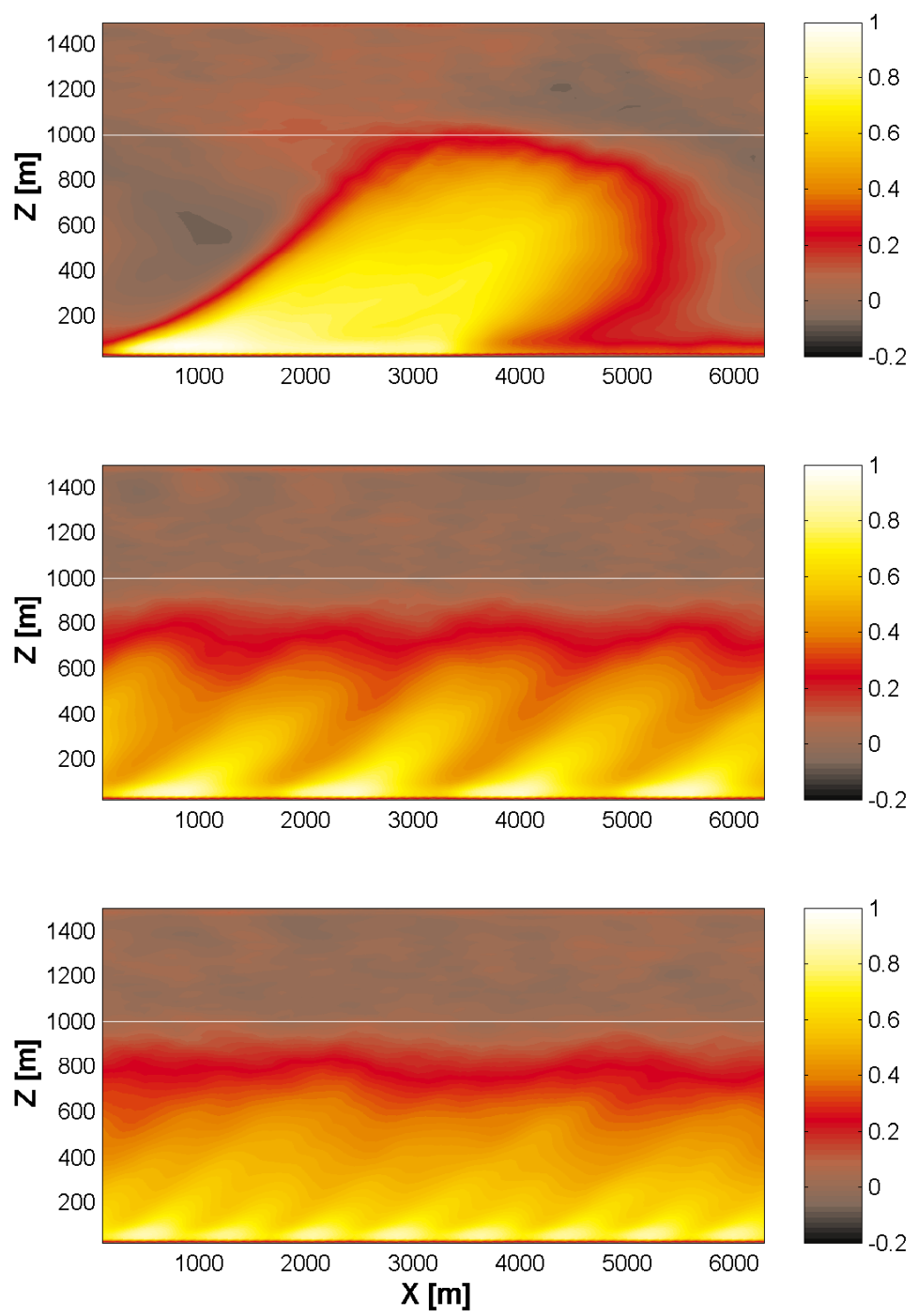

Fig. 5. As Fig. 4, but showing the correlation coefficient between vertical velocity and temperature $\left(\rho_{w \theta}\right)$.

sensible heat flux. Hence, the correlation between temperature and humidity is stronger over the main temperature source than over the main water source. This spatial inhomogeneity of $\rho_{\theta q}$ persists through the depth of the ABL, because of the strong vertical motion from the convection present over the hot patches in concert with the surface features being several times larger than the largest eddy responsible for the turbulent mixing. However, with the smaller surface patches the correlation between the scalars remains relatively strong across the horizontal direction, suggesting that both scalars are being removed from the surface in the same eddies. With these smaller patches the turbulence is efficiently mixing, or blending over, the variability in source areas.

From these statistical analyses of LES output over spatially variable surface fields it is apparent that it is possible to define the relative contributions of the sur- face sources/sinks and those at the capping inversion to the observed dissimilarity $[13,4,3]$ in the scaling of scalar mean fields and scalar variances. In fact, we see that second moments (e.g. fluxes) are affected to greater heights than are first moments (means). This is in agreement with many observations that mean water vapor and temperature fields behave more similarly than do their variances.

We explore the mixing more directly in Fig. 7, where we plot horizontal series of the $\rho_{\theta q}$ field taken at two different heights, one near the land surface $(z=9 \mathrm{~m})$ and one well into the mixed layer $(z=366$ $\mathrm{m})$. Note in the bottom panel that the scalars are near-perfectly correlated near the land for the small surface features, showing strong similarity of scalar transport, while the scalars are much less correlated for the case where the surface patches are larger than the depth of the ABL. In the upper panel we see the 

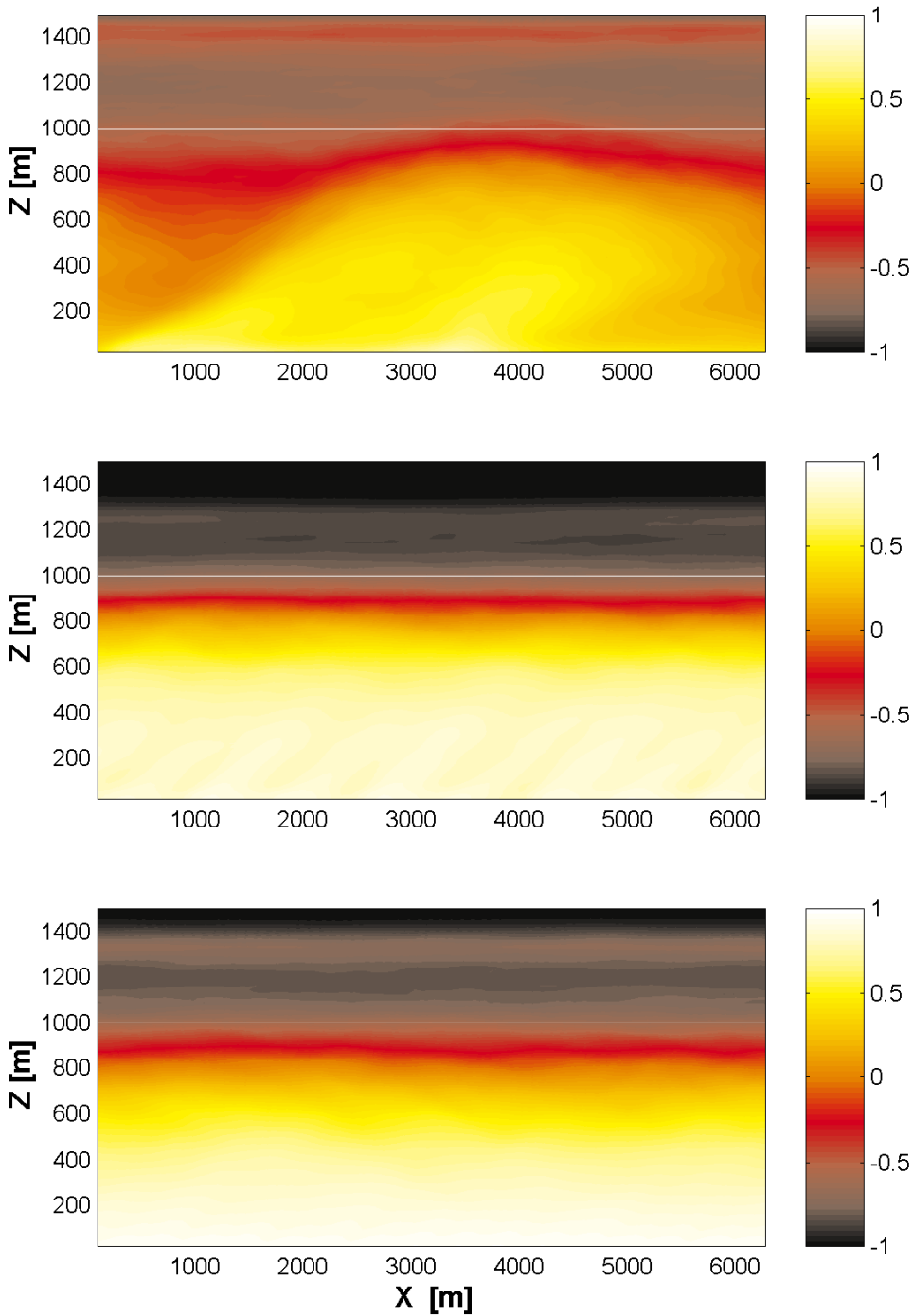

Fig. 6. As Fig. 4, but showing the correlation coefficient between vertical temperature and specific humidity $\left(\rho_{\theta q}\right)$.
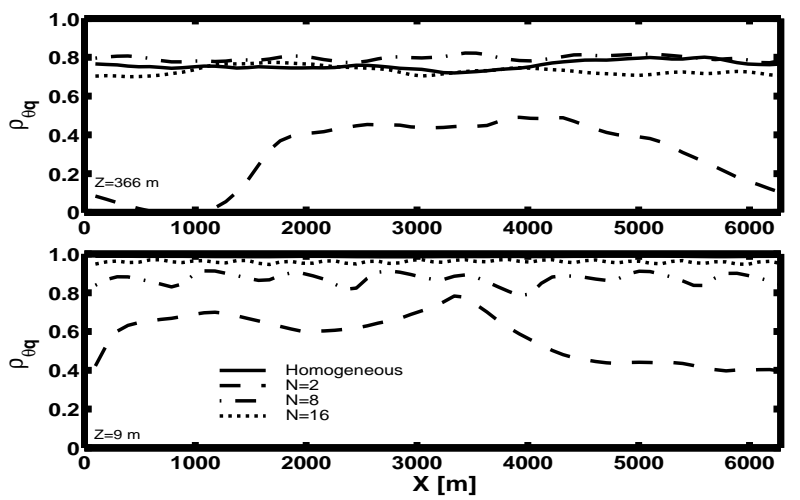

Fig. 7. Spatial series through Fig. 6 at two heights. The top panel shows $\rho_{\theta q}(x, z=366 \mathrm{~m})$ and the bottom panel shows $\rho_{\theta q}(x, z=9 \mathrm{~m})$.

effect of different eddy sources for the two scalars persisting to great heights for the large scale surface heterogeneity case.

\subsection{Blending of scalar fluctuation fields, deviation from homogeneous case}

So far we have seen evidence of turbulence blending with some insight into the mechanisms giving rise the spatial variability of the correlation coefficients. We now examine quantitatively the blending of the different correlation fields, using the homogeneous case as a reference. To accomplish this we introduce a new measure describing the extent to which the horizontal variability of the correlation coefficients in Figs. 4 and 5 (for each case) exceed that of the homogeneous case as a function of height.

The mean vertical profile of the correlation coefficient between variables $V_{1}$ and $V_{2}$ for case $N$ is defined as

$\tilde{\rho}_{v_{1} v_{2}, N}(z)=\frac{1}{L_{x}} \int \rho_{v_{1} v_{2}, N}(x, z) \mathrm{d} x$

and its horizontal standard deviation is 
$\sigma_{\tilde{\rho}_{v_{1} v_{2}, N}}(z)=\frac{1}{L_{x}}\left[\int\left(\rho_{v_{1} v_{2}, N}(x, z)-\tilde{\rho}_{v_{1} v_{2}, N}(z)\right)^{2} \mathrm{~d} x\right]^{1 / 2}$.

We study the vertical structure of the difference between this measure of spatial variability for case $N$ and that for the base case (subscript 0 )

$\Delta \sigma_{\tilde{\rho}_{v_{1} \nu_{2}, N}}(z)=\sigma_{\tilde{\rho}_{v_{1} v_{2}, N}}(z)-\sigma_{\tilde{\rho}_{v_{1} v_{2}, 0}}(z)$.

This direct measure of departure from homogeneity is presented in Fig. 8, for blending of $\rho_{w \theta}$ in the top panel and $\rho_{w q}$ in the bottom panel. From inspection it is clear that the boundary layer turbulence, for the stability regime studied, blends completely the latent heat fluxes for the $393 \mathrm{~m}$ surface features in the bottom $200 \mathrm{~m}$ of the $\mathrm{ABL}$, and for the $785 \mathrm{~m}$ surface case in the bottom 300 $\mathrm{m}$ or so of the ABL. For these two cases, the sensible heat fluxes are also well blended, but at somewhat greater heights. The sensible heat fluxes for the case with surface features larger than the ABL depth did not blend below the capping inversion. There is strong spatial variability in $\rho_{w \theta}$ throughout the depth of the ABL for this case. The water vapor fluxes are nearly blended for this $3142 \mathrm{~m}$ surface feature case in the bottom $400 \mathrm{~m}$ or so of the ABL, but we note greater spatial variability
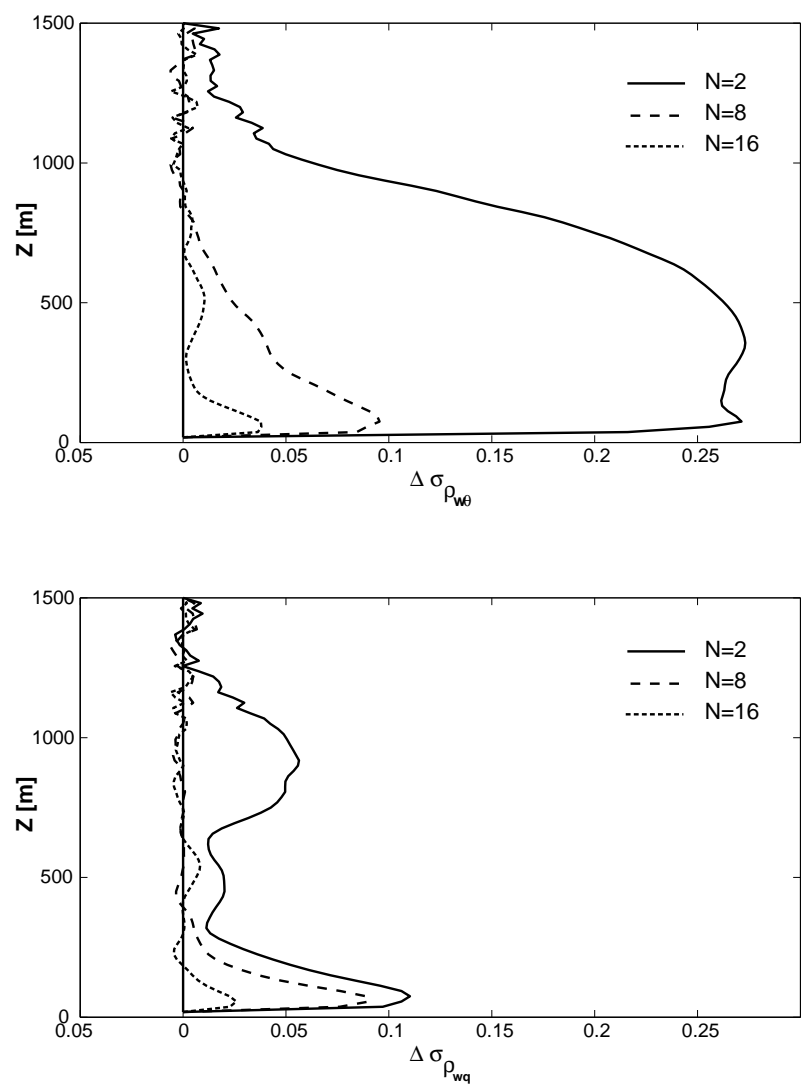

Fig. 8. Vertical profiles of the degree of inhomogeneity in the correlation fields compared to the homogeneous surface case. The $\rho_{w \theta}$ field is studied in the top panel and the $\rho_{w q}$ field in the bottom panel.

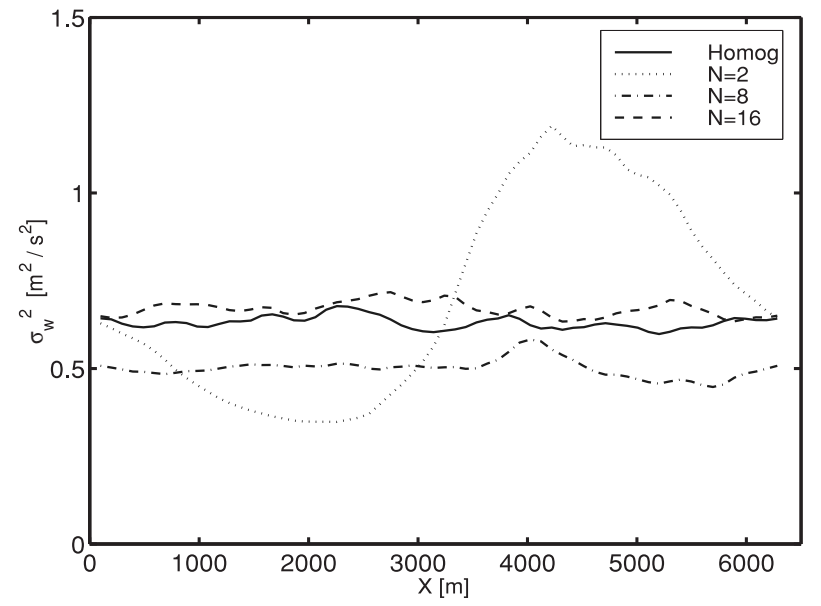

Fig. 9. Longitudinal series of the temporal vertical velocity variance for the three heterogeneous cases and the homogeneous case, all taken at a height just below the capping inversion.

near the capping inversion (i.e. around $Z=1000 \mathrm{~m}$ ). This variability in water vapor flux across the inversion is a byproduct of temperature effects on the vertical velocity fluctuations responsible for entrainment exchange across the inversion. Note from the mean humidity profile in Fig. 2 that a sharp specific humidity gradient exists across this layer. Hence, spatial variability in mixing (i.e. magnitude of $w^{\prime \prime}$ ) across this gradient will induce spatial variability in water vapor fluxes.

Fig. 9 shows the temporal variance of vertical velocity at the height of the capping inversion, averaged in $Y$ and plotted as a function of $X$. Note that this is a fairly horizontally homogeneous statistic for all cases except that with large surface patches. For the $L=3142 \mathrm{~m}$ case, there is evidence of greater mixing across the inversion in a region affected strongly by the localized source of sensible heat at the land surface. This localized region of strong mixing (due to temperature fluctuations creating vertical velocity fluctuations) is working on the sharp specific humidity gradients to create a localized region of higher $\rho_{w q}$ that is independent of the spatial variability of water vapor sources at the land surface.

\section{Conclusions}

The large eddy simulation (LES) of flow and transport of heat and water vapor in the atmospheric boundary layer (ABL) over spatially heterogeneous surfaces has been shown to be a useful tool for exploring the turbulent mixing phenomena responsible for the creation of a bending layer. Simulations were performed for three heterogeneous surfaces (with length scales smaller, similar, and greater than the ABL depth) and a homogeneous case with surface properties representing the average of the arid and wet fields employed in the heterogeneous cases. 
The mean scalar fields were shown to blend more efficiently (thinner blending layers) than the vertical scalar flux fields. Hence, separate blending layers should be defined for these two flow variables.

The water vapor flux field was shown to blend more efficiently than the sensible heat flux field, for similar surface differences (in terms of heat units). This difference between the blending of passive scalar fluxes (e.g. water vapor) and active scalar fluxes (e.g. temperature) is attributed to the role of temperature fluctuations in forcing the vertical velocity fluctuations through the buoyancy term, resulting in a greater vertical persistence for temperature sources. This explanation is shown clearly in the spatially resolved correlation fields, where water vapor appears to be carried more horizontally over the wet fields and ultimately carried upward in temperature driven eddies over downwind arid regions, while temperature sources are immediately convected upward in buoyant plumes, thus receiving less horizontal mixing. Under these strongly convective conditions, the concept of blending is shown to break down for both scalars as the length scale of the surface features becomes much larger than the ABL depth, and hence larger than the greatest transport eddies. This is in agreement with prevailing theory [35,22], and should be considered in the context of the strong vertical buoyancy forcing studied here - a difficult case for the blending mechanisms.

The introduction of a measure of the departure of the horizontal variability in correlation fields from the homogeneous case was used to define the blending heights for the different turbulent interactions (vertical velocity and water vapor; and vertical velocity and temperature) responsible for the fluxes. This measure vanishes at the height at which the correlation field converges to the homogeneous case.

\section{Acknowledgements}

The development of the original LES code was supported by a NASA Global Change Fellowship. We appreciate the considerable help with the fluid mechanics and code development from Wolfgang Kollmann and the insightful comments of Roger Shaw, both of UC Davis. The extension for scalar transport under density stratification and the performance of these simulations was supported by a faculty start-up grant from the University of Virginia. We are grateful for the contributions of four helpful, anonymous reviewers.

\section{References}

[1] Albertson JD. Large eddy simulation of land-atmosphere interaction, Ph.D. Thesis. University of California, Davis, 1996:185.
[2] Albertson JD, Parlange MB. Surface length scales and shear stress: Implications for land-atmosphere interaction over complex terrain. Water Resour Res, in press.

[3] Andreas EL, Hill RJ, Gosz J, Moore DI, Otto WD, Sarma AD. Statistics of surface layer turbulence over terrain with metre scale heterogeneity. Boundary Layer Meteorology 1998;86:379-408.

[4] Andren A, Brown AR, Graf J, Mason PJ, Moeng C-H, Nieuwstadt FTM, Schumann U. Large-eddy simulation of a neutrally stratified boundary layer: A comparison of four computer codes. Q J R Meteorol Soc 1994;120:1457-84.

[5] Avissar R, Eloranta EW, Gurer K, Tripoli GJ. An evaluation of the large eddy simulation option of the regional modeling system in simulating a convective boundary layer - A FIFE case study. J Atmos Sci 1998;55:1109-30.

[6] Brutsaert W, Parlange MB. The unstable surface layer above forest: Regional evaporation and heat flux. Water Resour Res 1992;28:3129-34.

[7] Brutsaert W, Parlange MB, Gash JHC. Neutral humidity profiles in the boundary layer and regional evaporation from sparse pine forest. Annales Geophysicae 1989;7:623-30.

[8] Canuto C, Hussaini MY, Quarteroni A, Zang TA. Spectral methods in fluid dynamics. Springer series in computational physics. Berlin: Springer, 1988:557.

[9] Claussen M. Estimation of areally averaged surface fluxes. Boundary-Layer Meteorology 1991;54:387-410.

[10] Deardorff JW. Preliminary results from numerical integrations of the unstable planetary boundary layer. J Atmos Sci 1970;27:120911.

[11] Deardorff JW. Convective velocity and temperature scales for the unstable planetary boundary layer and Rayleigh convection. J Atmos Sci 1970;27:1211-1213.

[12] Deardorff JW. Numerical investigation of neutral and unstable planetary boundary layers. J Atmos Sci 1972;29:91-115.

[13] De Bruin HAR, Bink NJ, Kroon LJM. Fluxes in the surface layer under advective conditions. In: Schmuggee T, Andre JC, editors. Land Surface Evaporation: Measurement and Parameterization 1991:157-69.

[14] Galperin B, Orszag SA, editors. Large eddy simulation of complex engineering and geophysical flows. Cambridge: Cambridge University Press, 1993:600.

[15] Garratt JR. The internal boundary layer - A review. Boundary Layer Meteorology 1990;50:171-203.

[16] Gao S, Leslie DC. Accuracy comparison of Adams-Bashforth and leapfrog in the context of large eddy simulation. Comm Appl Num Anal 1990;6:1-5

[17] Haltiner GJ, Williams RT. Numerical prediction and dynamic meteorology. New York: Wiley, 1980:477.

[18] Hechtel LM, Moeng C-H, Stull RB. The effects of nonhomogeneous surface fluxes on the convective boundary layer: A case study using large-eddy simulation. J Atmos Sci 1990;47:1722-41.

[19] Kaimal JC, Finnigan JJ. Atmospheric boundary layer flows. Oxford: Oxford University Press, 1994:289.

[20] Launder BE. Second moment closure: Present ... and future? Int J Heat Fluid Flow 1989;10:282-300.

[21] Leonard A. Energy cascade in large-eddy simulations of turbulent fluid flows. Advances in Geophysics 1974;18A:237-49.

[22] Mahrt L. The bulk aerodynamic formulation over heterogeneous surfaces. Boundary Layer Meteorology 1996;78:87-119.

[23] Mason PJ. The formation of areally averaged roughness lengths, Q J R Meteorol Soc 1988;114:399-420.

[24] Mason PJ, Callen NS. On the magnitude if the subgrid-scale eddy coefficient in large-eddy simulations of turbulent channel flow. J Fluid Mech 1986;162:439-62.

[25] Moeng C-H. A large eddy simulation for the study of planetary boundary layer turbulence. J Atmos Sci 1984;41:2052-62.

[26] Moeng C-H, Wyngaard JC. Statistics of conservative scalars in the convective boundary layer. J Atmos Sci 1984;41:3161-9. 
[27] Moin P, Reynolds WC, Ferzinger JH. Large eddy simulation of incompressible turbulent channel flow. Department of Mechanical Engineering, Stanford University Report 1978;TF-12.

[28] Monin AS, Obukhov AM. Basic laws of turbulent mixing in the ground layer of the atmosphere. Tr Geofiz Inst Akad Nauk SSSR 1954;151:163-87.

[29] Nieuwstadt FTM, Mason PJ. Moeng C-H, Schumann U. Largeeddy simulation of the convective boundary layer: A comparison of four computer codes. Turbulent Shear Flows 1991;8:343-67.

[30] Parlange MB, Brutsaert W. Regional shear stress of broken forest from radiosonde wind profiles in the unstable surface layer. Boundary Layer Meteorology 1993;64:355-69.

[31] Parlange MB, Eichinger WE, Albertson JD. Regional evaporation into the atmospheric boundary layer. Reviews of Geophysics 1995;33:99-124.

[32] Parlange MB, Katul GG. Watershed scale shear stress from tethersonde wind profile measurements under near neutral and unstable atmospheric stability. Water Resour Res 1995;31:961-8.

[33] Perrier A, Tuzet A. Land surface processes: Description, theoretical approaches, and physical laws underlying their measurement. In: Schmugge TJ, Andrè J-C., editors. Land surface evaporation. Berlin: Springer, 1991:145-55.
[34] Philip JR. Blending and internal boundary-layer heights and shear stress. Boundary Layer Meteorology 1997;84:85-98.

[35] Raupach MR. Vegetation-atmosphere interaction in homogeneous and heterogeneous terrain: some implications for mixedlayer dynamics. Vegetatio 1991;91:105-20.

[36] Shen SH, Leclerc MY. How large must surface inhomogeneities be before they influence the convective boundary layer structure A case study. Q J R Meteorol Soc 1995;121:1209-28.

[37] Schmidt H, Schumann U. Coherent structures of the convective boundary layer derived from large-eddy simulations, J Fluid Mech 1989;200:511-62.

[38] Smagorinsky J. General circulation experiments with the primitive equations, Part 1: the basic experiment. Monthly Wea Rev 1963;91:99-164.

[39] Stull R. An introduction to boundary layer meteorology. Dordrecht: Kluwer Academic Press, 1988:666.

[40] Sutton OG. Wind structure and evaporation in a turbulent boundary layer. Proc Roy Soc London A 1934;146:701-22.

[41] Wood N, Mason PJ. The influence of static stability on the effective roughness length for momentum and heat transfer. Q J R Meteorol Soc 1991;117:1025-56. 